\title{
Appraising the Value of Digital Health Technologies From the Managed Care Perspective: Insights for Evidence Assessment and Reimbursement in the United States
}

Thomas Walters ${ }^{1 *}$, PharmD, MBA, MS; Benjamin Parcher ${ }^{1 *}$, PharmD; Alexander Kravetz ${ }^{2}$, PharmD; Chelsey Campbell ${ }^{1}$, PharmD, MBA, MS; Manasee Shah ${ }^{1}$, MPH

\footnotetext{
${ }^{1}$ Xcenda, AmerisourceBergen Consulting Services, Palm Harbor, FL, United States

${ }^{2}$ Optum Labs, Cambridge, MA, United States

*these authors contributed equally
}

\section{Corresponding Author:}

Thomas Walters, PharmD, MBA, MS

Xcenda, AmerisourceBergen Consulting Services

4114 Woodlands Pkwy

Palm Harbor, FL, 34685

United States

Phone: 12629515207

Fax: 17277714100

Email: thomas.walters@xcenda.com

\section{Abstract}

Background: Digital health technologies (DHTs) have accelerated in both number and utility in recent years, prompting managed care organizations (MCOs) to define the segment's value and role in improving the health care of their members. In this context, many technology manufacturers have initiated clinical trials to generate evidence supporting DHTs; however, limited guidance remains on how MCOs formally evaluate these products.

Objective: To understand how medical and pharmacy directors assess the value of DHTs in the United States and to identify best practices for supporting their reimbursement determinations.

Methods: Medical and pharmacy directors within Xcenda's Managed Care Network (MCN) were invited to complete a 10-part, web-based questionnaire. Respondents were asked to grade their organization's current demand and coverage policy of 9 distinct categories of DHTs. Eleven major disease classes were evaluated based on the potential impact DHTs can have for addressing unmet needs. Specific evidence requirements for reimbursement of DHTs were then proposed and rated. Finally, strategies for manufacturers to interface with MCOs were examined.

Results: In total, 37 pharmacy directors (60.7\%) and 24 medical directors (39.3\%) completed the questionnaire. The respondents' MCOs cover approximately 180 million lives in the United States, with a mix of national (34.4\%) and regional (65.6\%) plans. Of the 9 technologies evaluated, mobile apps (80.3\%), fitness trackers (60.7\%), and medication adherence platforms $(57.4 \%)$ scored the highest in demand for implementation as a covered benefit. Diabetes (88.5\%) and cardiovascular disease (86.9\%) were ranked highest in potential impact for DHTs to address unmet needs. Peer-reviewed literature (96.7\%) was rated as the most important evidence resource in evaluating the DHTs, followed by real world analysis (95.1\%), and cost-effectiveness models (78.7\%). Clinical benefit (96.7\%) was the top evidence criteria selected for coverage determination. Advisory board meetings (70.5\%), continuing education sessions (57.4\%), and dedicated peer-reviewed journals for DHTs (55.7\%) were identified as the preferred communication strategies between manufacturers and MCOs.

Conclusions: MCOs are actively evaluating a wide range of DHTs in a variety of disease states. Traditional appraisal strategies used in the evaluation of medical devices and pharmaceutical products are seen to also apply in evaluating DHTs. Respondents indicated that more robust evidence communication strategies with technology manufacturers and MCOs are needed for coverage decision making.

(iproc 2016;2(1):e14) doi: 10.2196/iproc.6031 


\section{KEYWORDS}

digital health technology; value; managed care; evidence assessment; reimbursement

This poster was presented at the Connected Health Symposium is displayed as an image in Figure 1 and as a PDF in Multimedia 2016, October 20-21, Boston, MA, United States. The poster Appendix 1.

Figure 1. Poster.



\section{Multimedia Appendix 1}

Poster.

[PDF File (Adobe PDF File), 511KB-Multimedia Appendix 1]

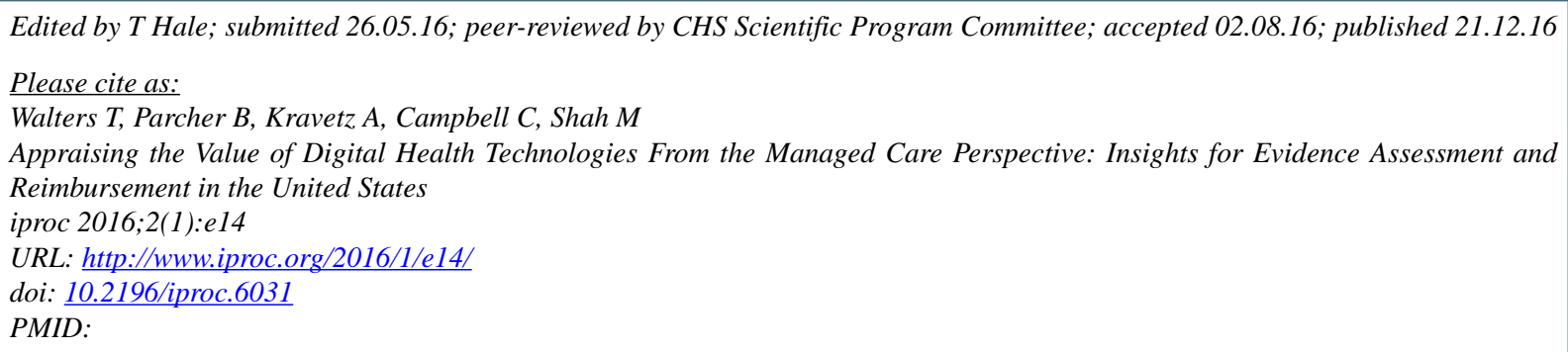

(C)Thomas Walters, Benjamin Parcher, Alexander Kravetz, Chelsey Campbell, Manasee Shah. Originally published in Iproceedings (http://www.iproc.org), 21.12.2016. This is an open-access article distributed under the terms of the Creative Commons Attribution License (http://creativecommons.org/licenses/by/2.0/), which permits unrestricted use, distribution, and reproduction in any medium, provided the original work, first published in Iproceedings, is properly cited. The complete bibliographic information, a link to the original publication on http://www.iproc.org/, as well as this copyright and license information must be included. 